PESQUIMAT, Revista de la Fac.CC.MM. de la

UNIVERSIDAD NACIONAL MAYOR. DESAN MARCOS

Vol.IV, $N^{\circ} 1$,pag.31-38,LIMA-PERÚ. Julio 2001

\title{
INVARIANZA TOPOLÓGICA DEL ÍNDICE DE INTERSECCIÓN
}

\author{
Renato Benazic Tomé ${ }^{1}$
}

\begin{abstract}
Vamos a considerar campos vectoriales holomorfos con singularidad aislada dicrítica, cuyos transformados estrictos no presentan singularidades on el divisor. En estas condiciones, es posible asociar a cada hoja del transformado estricto, un índice de intersccción con cl divisor. El resultado principal de cste trabajo, esं probar que este índice cs un invariante topológico.
\end{abstract}

\section{INTRODUCCIÓN}

Sea $\mathcal{M}^{n}$ una variedad analítica compleja de dimensión $n$ y consideremos en ella una foliación analítica singular por curvas. Esto significa que en cualquier punto $p \in \mathcal{M}^{n}$ la foliación es generada por el campo vectorial holomorfo

$$
Z=\sum_{i=1}^{n} Z_{i} \frac{\partial}{\partial z_{i}}, Z_{1}, Z_{2}, \ldots, Z_{n} \in \mathcal{O}_{n, p} ; \text { y } \quad \text { m.c.d. }\left(Z_{1}, Z_{2}, \ldots, Z_{n}\right)=1
$$

en donde $\mathcal{O}_{n, p}$ es el anillo de gérmenes de las funciones analíticas en $p$. En lo sucesivo, denotaremos por $\mathcal{F}_{Z}$ a esta foliación, diremos que el campo $Z$ genera la foliación $\mathcal{F}_{Z}$ y las funciones $Z_{i}$ serán llamadas componentes de $Z$. El lector interesado en conocer detalles de la teoría de funciones analíticas de varias complejas, deberá consultar [7] y para los que deseen profundizar en la teoría de las variedades analíticas complejas, recomendamos [9]. Sea $p \in \mathcal{M}^{n}$ y consideremos una carta. $(U, \phi)$ de $\mathcal{M}^{n}$ alrededor del punto $p$ tal que $\phi(p)=0 \in \mathbb{C}^{n}$, claramente. $Z_{i} \circ \phi^{-1}$ es una función analítica de varias variables complejas definida en una vecindad del origen y por lo tanto, ella tiene un desarrollo en

\footnotetext{
${ }^{1}$ Universidad Nacional Mayor de San Marcos. Facultad de Ciencias Matemáticas.
} 
series de potencias

$$
Z_{i} \circ \phi^{-1}=\sum_{k \geq 0} Z_{i}^{k}, \quad 1 \leq i \leq n
$$

donde $\operatorname{los} Z_{i}^{k}$ son polinomios homogéneos de grado $k$ en $n$ variables complejas. El orden de $Z_{i} \circ \phi^{-1}$ en el $0 \in \mathbb{C}^{n}$ es, por definición, el menor número entero $\nu_{i}$ tal que $Z_{i}^{k} \equiv 0$, para todo $k<\nu_{i}$ y $A_{i}^{\nu_{i}} \not \equiv 0$. No es dificil probar que el número $\nu_{i}$ es independiente de la elección de la carta $(U, \phi)$, por esta razón el entero $\nu_{i}$ es llamado el orden de $Z_{i}$ en $p$ y lo denotamos por $\operatorname{ord}_{p}\left(Z_{i}\right)$. La multiplicidad algebraica de la foliación $\mathcal{F}_{Z}$ (o del campo $Z$ ) en el punto $p \in \mathcal{M}^{n}$, denotada por $m_{p}\left(\mathcal{F}_{Z}\right)$ (o simplemente por $m_{p}(Z)$ ), es definida como el mínimo de los órdenes $\operatorname{ord}_{p}\left(Z_{i}\right)$. Un punto $p \in \mathcal{M}^{n}$ es llamado punto singular de la foliación $\mathcal{F}_{Z}$ (o del campo $Z$ ) si y sólo si $m_{p}(Z) \geq 1$, en caso contrario decimos que $p$ es un punto regular. El conjunto de todos los puntos singulares de la foliación $\mathcal{F}_{Z}$ será denotado por $\operatorname{Sing}\left(\mathcal{F}_{Z}\right)$. Un punto $p \in \mathcal{M}^{n}$ es llamado singularidad aislada de $Z$ si y sólo si $p \in \operatorname{Sing}\left(\mathcal{F}_{Z}\right)$ y existe una vecindad abierta $U \subseteq \mathcal{M}^{n}$ de $p$ tal que todos los elementos de $U-\{p\}$ son puntos regulares de $Z$. Sea $E: \tilde{\mathcal{M}}^{n} \rightarrow \mathcal{M}^{n}$ el blowup centrado en el punto $p \in \operatorname{Sing}\left(\mathcal{F}_{Z}\right)$. Entonces existe una única manera de extender $E^{*}\left(\mathcal{F}_{Z}-\{p\}\right)$ a una foliación analítica singular $\tilde{\mathcal{F}}_{Z}$ sobre una vecindad del espacio proyectivo $\mathbb{C} P(n-1)=E^{-1}(p) \subset \tilde{\mathcal{M}}^{n}$, con conjunto singular de codimension mayor o igual que 2 . En este caso decimos que $\tilde{\mathcal{F}}_{Z}$ es el transformado estricto de $\mathcal{F}_{Z}$ por $E$. El lector interesado en mayores detalles sobre el tema, puede consultar las referencias [6], [8], [2] y [4].

Decimos que $p$ es una singularidad no dicrítica de $\mathcal{F}_{Z}$ si y sólo si $E^{-1}(p)$ es invariante por $\tilde{\mathcal{F}}_{Z}$, es decir, $E^{-1}(p)$ es unión de hojas y singularidades de $\tilde{\mathcal{F}}_{Z}$. En caso contrario, $p$ es llamado singularidad dicrítica. El conjunto de las foliaciones analíticas por curvas sobre $\mathcal{M}^{n}$ con una singularidad dicrítica aislada, será denotado por $\mathcal{D}^{n}$.

Sea $\mathcal{F}_{Z} \in \mathcal{D}^{n}$ tal que $m_{p}(Z)=\nu$. En [1] se demuestra que es posible asociar a $J_{p}^{\nu}(Z)$, el primer jet no nulo de $\mathcal{F}_{Z}$ en $p$, un polinomio homogéneo $P_{\nu-1}$ den $n$ variables, de grado $\nu-1$ tal que:

$$
J_{p}^{\nu}(Z)=: P_{\nu-1} R
$$

donde $R(z)=Z$ es el campo radial.

El polinomio $P_{\nu-1}$ define una hipersuperficie algebraica $S$ sobre el espacio proyectivo $E^{-1}(p)=\mathbb{C} P(n,-1)$ :

$$
S=\left\{\left[z_{1} ; \ldots ; z_{n}\right] \in \mathbb{C} P(n-1): P_{\nu-1}\left(z_{1}, \ldots, z_{n}\right)=0\right\}
$$


Observe que $S$ contiene todas las singularidades del transformado estricto $\tilde{\mathcal{F}}_{Z}$. La hipersuperficie $S$ tiene la siguiente interpretación geométrica: Si $\tilde{p} \in S-\operatorname{Sing}\left(\tilde{\mathcal{F}}_{Z}\right)$ entonces la hoja $\tilde{L}$ de $\tilde{\mathcal{F}}_{Z}$ que pasia por $\tilde{p}$ es tangente al espacio proyectivo $E^{-1}(p)$ y si $\tilde{p} \in E^{-1}(p)-S$ entonces $\tilde{L}$ es transversal a $E^{-1}(p)$.

Un caso importante ocurre cuando $\operatorname{Sing}\left(\tilde{\mathcal{F}}_{Z}\right)=\emptyset$. es decir, cuando el transformado estricto de $\mathcal{F}_{Z}$ no tiene singularidades en $\tilde{\mathcal{M}}^{n}$. Denotaremos por $\mathcal{D}_{0}^{n}$ al conjunto de tales foliaciones. Observe que, en este caso, cada $\tilde{p} \in E^{-1}(p)$ es un punto regular de $\tilde{\mathcal{F}}_{Z}$. Por lo tanto. es posible definir el indice de intersección $i_{\tilde{p}}\left(E^{-1}(p), \tilde{L}\right)$ entre la hoja $\tilde{L}$ de $\tilde{\mathcal{F}}_{Z}$ que pasa por $\tilde{p} \in E^{-1}(p)$ y el espacio provectivo $E^{-1}(p)$. El resultado principal del presente trabajo es que el índice de intersección es un invariante topológico.

\section{EL ÍNDICE DE INTERSËCCIÓN}

Sea $\mathcal{F}_{Z} \in \mathcal{D}_{0}^{n}$ generado por el campo vectorial holomorfo $Z$. sin pérdida de generalidad, podemos suponer que la singularidad aislada dicrítica es el $0 \in \mathbb{C}^{n}$. Sabemos que cualquier punto $\tilde{p} \in E^{-1}(0)=$ $\mathbb{C} P(n-1)$ es un punto regular de la foliación $\tilde{\mathcal{F}}_{Z}$. Sea $\tilde{L}$ la hoja de $\tilde{\mathcal{F}}_{Z}$ que pasa por $\tilde{p}$. Desde que $E^{-1}(0)$ y $\tilde{L}$ son subvariedades analíticas de $\mathbb{C}^{n}$ de dimensiones complementarias (ver [5]) y $\tilde{p} \in E^{-1}(0) \cap \tilde{L}$. podemos definir el indice de intersección $i_{\tilde{p}}\left(E^{-1}(0), \tilde{L}\right)$ de la hoja $\tilde{L}$ con el espacio proyectivo $E^{-1}(0)$ en el punto $\tilde{p}$. En efecto, podemos suponer, sin pérdida de generalidad, que $\tilde{p}$ está en la carta de blow-11p en donde $E$ se expresa como:

$$
E\left(y_{1}, \ldots, y_{n}\right)=\left(y_{1} y_{n}, \ldots, y_{n-1} y_{n}, y_{n}\right)=\left(z_{1} \ldots, z_{n}\right)
$$

Desde que $\tilde{p}=\left(y_{1}^{0}, \ldots, y_{n-1}^{0}\right.$. 0$)$ es un punto regular. la hoja $\tilde{L}$ puede ser localmente parametrizada por una función analítica

$$
\tilde{\alpha}=\left(\tilde{\alpha}_{1}, \ldots, \tilde{\alpha}_{n}\right):\left(\mathbb{D}_{\uparrow}, 0\right) \rightarrow(\tilde{L}, \tilde{p})
$$

donde $\mathbb{D}_{\epsilon}=\{T \in \mathbb{C}:|T|<\epsilon\}$, tal que

$$
\begin{aligned}
& \tilde{\alpha}^{\prime}(T)=\tilde{Z}(\tilde{\alpha}(T)), \quad \forall T \in \mathbb{D}_{\epsilon} \\
& \tilde{\alpha}(0)=\tilde{p} .
\end{aligned}
$$

Desde que $E^{-1}(0)=\left\{y_{n}=0\right\}$, podemos definir: 


$$
i_{\tilde{p}}\left(E^{-1}(0), \tilde{L}\right)=\operatorname{ord}_{0}\left(\tilde{\alpha}_{n}\right)
$$

es decir, $i_{\tilde{p}}\left(E^{-1}(0), \tilde{L}\right)=m \Longleftrightarrow \tilde{\alpha}_{n}(T)=T^{m} \tilde{\varepsilon}_{n}(T)$ donde $\tilde{\varepsilon}$ es una función analítica y $\tilde{\varepsilon}_{n}(0) \neq 0$. Es claro que $i_{\tilde{p}}\left(E^{-1}(0), \tilde{L}\right) \geq 1, \forall \tilde{p} \in$ $E^{-1}(0)$.

El índice de intersección puede ser geométricamente interpretado como el número de puntos de intersección de $\tilde{L}$ con una pequeña traslación del espacio proyectivo $\left\{y_{n}=\delta\right\}$, con $\delta \neq 0$. Para mavor información sobre las propiedades del índice de intersección entre subvariedades analíticas de dimensiones complementarias, el lector debe consultar [5].

Observe que si $\tilde{p} \in E^{-1}(0)$ entonces $\tilde{\alpha}_{n}^{\prime}(0)=P_{\nu-1}\left(y_{1}^{0}, \ldots, y_{n-1}^{0}, 1\right)$ entonces concluimos que $i_{\tilde{p}}\left(E^{-1}(0), \tilde{L}\right)=1$ si y sólo si $\tilde{p} \in E^{-1}(0)-S$ si y sólo si $\tilde{L}$ es transversal a $E^{-1}(0)$ en $\tilde{p}$.

\section{MULTIPLICIDAD DE UN CAMPO VECTORIAL A LO LARGO DE UNA VARIEDAD INVARIANTE}

Nuestro objetivo es probar que el índice de intersección definido en la sección anterior es un invariante topológico. La principal herramienta para probar este resultado es el uso de la invarianza topológica de la multiplicidad de un campo vectorial a lo largo de una subvariedad analítica invariante. Las pruebas de los resultados dados en esta sección, el lector las puede encontrar en [3].

Sea $Z$ un germen en $0 \in \mathbb{C}^{n}$ de un campo vectorial holomorfo y $L$ un germen en $0 \in \mathbb{C}^{n}$ de una subvariedad analítica irreducible invariante por $Z$ y de $\operatorname{dim}_{\mathbb{C}} L=1$. Si $B$ es una bola suficientemente pequeña centrada en $0 \in \mathbb{C}^{n}$ tal que $B \cap L$ es conexo, entonces $B \cap L$ es homeomorfo a un disco bidimensional $\mathbb{D}_{\epsilon}=\{T \in \mathbb{C}:|T|<\epsilon\}$. Tal homeomorfismo puede ser realizado, por ejemplo, mediante una parametrización de Puiseaux $\alpha:\left(\mathbb{D}_{\epsilon}, 0\right) \rightarrow(B \cap L, 0)$, de $B \cap L$, donde $\alpha(T)=\left(\alpha_{1}(T), \ldots, \alpha_{n}(T)\right), \alpha_{j}(T)=T^{m_{j}} \varepsilon_{j}(T) \operatorname{con} \varepsilon_{j}(0) \neq 0$ o $\varepsilon_{j}=0$ y $m_{j} \in \mathbb{Z}^{+}, \forall 1 \leq j \leq n$.

Bajo estas condiciones, $\left.Z\right|_{B \cap L}$ puede ser considerado como un germen de un campo vectorial real con singularidad aislada, luego su índice en $0 \in \mathbb{C}^{n}$ esta bien definido.

Definición 1. Sean $Z, L$ y $B$ como antes. Definimos la Multiplicidad de $Z$ a lo largo de $L$ en $0, m_{0}(Z, L)$ como el indice de $\left.Z\right|_{B \cap L}$ en $0 \in \mathbb{C}^{n}$. considerado como un campo vectorial real en $B \cap L$. 
La siguiente Proposición establece que el número $m_{0}(Z, L)$ puede ser calculado en términos de una parametrización de Puiseaux $\alpha$ de $L$ :

Proposición 1. Con las notaciones anteriores, existe un único campo vectorial holomorfo $X$ en $\mathbb{D}_{\epsilon}$ con $X(0)=0$ tal que:

$$
Z(\alpha(T))=X(T) \alpha^{\prime}(T), \quad \forall T \in \mathbb{D}_{\epsilon}
$$

Además, si $X(T)=T^{m} \beta(T) \frac{\partial}{\partial T}\left(m \in \mathbb{Z}^{+}\right)$, con $\beta(0) \neq 0$, entonces

$$
m_{0}(Z, L)=m \text {. }
$$

Definición 2. Sean $\mathcal{F}_{Z}$ y $\mathcal{F}_{Z^{\prime}}$ dos gérmenes de foliaciones en $0 \in C^{n}$. Decimos que $\mathcal{F}_{Z}$ y $\mathcal{F}_{Z^{\prime}}$ son topológicamente equivalentes, lo cual será denotado por $\mathcal{F}_{Z} \sim_{\text {top }} \mathcal{F}_{Z^{\prime}}$ (o simplemente por $Z \sim_{\text {top }} Z^{\prime}$ ) si y sólo si existe $h$ germen de homeomorfismo en $0 \in \mathbb{C}^{n}$.tal que $h^{*}\left(\mathcal{F}_{Z^{\prime}}\right)=\mathcal{F}_{Z}$. Es decir, $L \in \mathcal{F}_{Z} \Longrightarrow h[L] \in \mathcal{F}_{Z^{\prime}}$. h es llamado conjugación topológica.

Sean $\mathcal{F}_{Z}$ y $\mathcal{F}_{Z^{\prime}}$ dos gérmenes de foliaciones con singularidad aislada en $0 \in \mathbb{C}^{n}$, tales que $\mathcal{F}_{Z} \sim_{\text {top }} \mathcal{F}_{Z^{\prime}}$ por un homeomorfismo $h: U \rightarrow U^{\prime}$ donde $U$ y $U^{\prime}$ son vecindades abiertas del 0 . Sea $L$ una subvariedad analítica, irreducible, invariante por $Z$ con singularidad aislada en $0 \in$ $\mathbb{C}^{n}$ y $\operatorname{dim}_{\mathbb{C}} L=1$. Podemos suponer que $L \cap U$ es conexa. Bajo estas condiciones, se sigue que $L-\{0\}$ es una hoja de $\mathcal{F}_{Z}$ y por el teorema de Remmert-Stein (ver [7]), tenemos que $L^{\prime}=h[L]$ es una subvariedad analítica, irreducible, invariante por $Z^{\prime}$ con $\operatorname{dim}_{\mathbf{C}} L^{\prime}=1$. Por lo tanto, $m_{0}\left(Z^{\prime}, L^{\prime}\right)$ esta bien definido. Tenemos el siguiente resultado.

Proposición 2. La multiplicidad de $Z$ a lo largo de $V$ es un invariante topológico. Más específicamente, sean $L$ y $L^{\prime}$ como antes. Si $\mathcal{F}_{Z} \sim_{\text {top }}$ $\mathcal{F}_{Z^{\prime}}$ entonces $m_{0}(Z, L)=m_{0}\left(Z^{\prime}, L^{\prime}\right)$.

\section{EL RESULTADO PRINCIPAL}

Retornando a las foliaciones dicríticas, probaremos que existe una relación entre el índice de intersección y la multiplicidad a lo largo de una subvariedad invariante. En efecto, sea $\mathcal{F}_{Z} \in \mathcal{D}_{0}^{n}$, con $m_{0}\left(\mathcal{F}_{Z}\right)=\nu$ y $\tilde{p} \in E^{-1}(0)=\mathbb{C} P(n-1)$, podemos suponer, sin pérdida de generalidad, que $\tilde{p}=\left(y_{1}^{0}, \ldots, y_{n-1}^{0}\right)$. Sea $\tilde{U}$ vecindad de $\tilde{p}$ tal que $\left.\tilde{L}\right|_{\tilde{U}} \cap E^{-1}(0)=\{\tilde{p}\}$, donde $\tilde{L}$ es la hoja de $\tilde{\mathcal{F}}_{Z}$ que pasa por $\tilde{p}$. Si $\epsilon>0$ es suficientemente pequeño, entonces $\left.\tilde{L}\right|_{\tilde{U}}$ puede ser parametrizado por la función analítica 


$$
\tilde{\alpha}=\left(\tilde{\alpha}_{1}, \ldots, \tilde{\alpha}_{n}\right):\left(\mathbb{D}_{\epsilon}, 0\right) \rightarrow(\tilde{l}, \tilde{p})
$$

la cual satisface

$$
\begin{aligned}
& \tilde{\alpha}^{\prime}(T)=\tilde{Z}(\tilde{\alpha}(T)), \quad \forall T \in \mathbb{D}_{\epsilon} \\
& \tilde{\alpha}(0)=\tilde{p} .
\end{aligned}
$$

Si $\tilde{\alpha}_{n}(T)=T^{m} \tilde{\varepsilon}_{n}(T)$ con $\tilde{\varepsilon}_{n}(0) \neq 0$ y $m \in \mathbb{Z}^{+}$, se sigue de la definición de índice de intersección que $i_{\tilde{p}}\left(E^{-1}(0), \tilde{L}\right)=m$. Sea $U$ vecindad de $0 \in \mathbb{C}^{n}$ tal que $E^{-1}[U-\{0\}] \subseteq \tilde{U}$ y $E\left[\left.\tilde{L}\right|_{\tilde{U}}-\{\tilde{p}\}\right]=L-\{0\}$. Entonces $L$ es una subvariedad analítica, irreducible de $U$, invariante por $\mathbb{Z}$, con singularidad aislada en 0 y $\operatorname{dim}_{\mathbb{C}} L=1$. Definimos $\alpha:\left(\mathbb{D}_{\epsilon}, 0\right) \rightarrow(L, 0)$ como:

$$
\alpha(T)= \begin{cases}E \tilde{\alpha}(T)=\tilde{Z}(\tilde{\alpha}(T)), & T \neq 0 \\ 0, & T=0\end{cases}
$$

se sigue que $\alpha$ es una parametrización de Puiseaux de $L$. Desde que $\tilde{Z}=\frac{E^{*} Z}{y_{n}^{\nu}}($ ver $[1])$, tenemos que:

$$
\begin{aligned}
\tilde{\alpha}^{\prime}(T) & =\tilde{Z}(\tilde{\alpha}(T))=\frac{D E^{-1} \circ E(\tilde{\alpha}(T)) Z \circ E(\tilde{\alpha}(T))}{\tilde{\alpha}_{n}(T)^{\nu}} \\
& =\frac{D E^{-1}(\alpha(T)) Z(\alpha(T))}{\tilde{\alpha}_{n}(T)^{\nu}}=\frac{X(T)}{\tilde{\alpha}_{n}(T)^{\nu}}\left(E^{-1} \circ \alpha\right)^{\prime}(T) \\
& =\frac{X(T)}{\tilde{\alpha}_{n}(T)^{\nu}} \tilde{\alpha}^{\prime}(T)
\end{aligned}
$$

donde $X$ es el campo vectorial holomorfo de la Proposición 1 y $T \in$ $\mathbb{D}_{\epsilon}-\{0\}$.

Concluimos que $X(T)=\tilde{\alpha}_{n}(T)^{\nu}=T^{m \nu} \beta(T)^{\nu}$, con $\beta(0) \neq 0$. De esta manera, hemos demostrado el siguiente:

Lema. Si $\mathcal{F}_{Z} \in \mathcal{D}_{0}^{n}, \tilde{p} \in E^{-1}(0)$, L y $\tilde{L}$ como antes. En las condiciones mencionadas, tenemos que

$$
m_{0}(Z, L)=m_{0}(Z) i_{\tilde{p}}\left(E^{-1}(0), \tilde{L}\right) .
$$

Ahora podemos probar que la multiplicidad algebraica de una foliación en $\mathcal{D}_{0}^{n}$, y el índice de intersección son invariantes topológicos. En efecto, sean $\mathcal{F}_{Z}$ y $\mathcal{F}_{Z^{\prime}}$ dos elementos de $\mathcal{D}_{0}^{n}$ tales que $\mathcal{F}_{Z} \sim_{\text {top }} \mathcal{F}_{Z^{\prime}}$ por un germen de homeomorfismo $h$. Sea $\tilde{p} \in E^{-1}(0)-S$, donde $S$ es la hipersuperficie de tangencia de $\mathcal{F}_{Z}$, desde que $\tilde{L}$, la hoja de 
$\tilde{\mathcal{F}}_{Z}$ que pasa por $\tilde{p}$, es transversal al espacio proyectivo $E^{-1}(0)$, tenemos que $i_{\tilde{p}}\left(E^{-1}(0), \tilde{L}\right)=1$. Consideremos $\tilde{U}$ vecindad de $\tilde{p}$ tal que $\tilde{L}{ }_{\tilde{U}} \cap E^{-1}(0)=\{\tilde{p}\}$ y $U$ vecindad de $0 \in \mathbb{C}^{n}$ tal que $E^{-1}[U-\{0\}] \subseteq \tilde{U}$. Si $L=\overline{E\left[\left.\tilde{L}\right|_{\tilde{U}}-\{\tilde{p}\}\right]}$ entonces $L-\{0\}$ es una hoja de $\mathcal{F}_{Z}$ y $L$ es una subvariedad analítica, irreducible de $U$, invariante por $Z$ y $\operatorname{dim}_{\mathbb{C}} L=1$.

De la definición de $h$, se sigue que $h[L-\{0\}]$ es una hoja de $\mathcal{F}_{Z^{\prime}}$. Sea $U^{\prime}=h[U]$ y $L^{\prime}=\overline{h[L-\{0\}]}$, se sigue del teorema de Remmert-Stein que $L^{\prime}$ es una subvariedad analítica, irreducible de $U^{\prime}$, con singularidad aislada en $0 \in \mathbb{C}^{n}$ y $\operatorname{dim}_{\mathbb{C}} L^{\prime}=1$. Desde que $L^{\prime}-\{0\} \in \mathcal{F}_{Z^{\prime}}$ entonces $E^{-1}[U-\{0\}] \in \mathcal{F}_{Z^{\prime}}$. Por lo tanto $\tilde{L}^{\prime}=\overline{E^{-1}[U-\{0\}]}$ esta contenido en una hoja de $\tilde{\mathcal{F}_{Z^{\prime}}}$ y $\tilde{L}^{\prime} \cap E^{-1}(0)=\left\{p^{\prime}\right\}$.

Bajo estas condiciones, del Lema anterior tenemos que $m_{0}(Z . L)=$ $m_{0}(Z)$ y de la invarianza topológica de la multiplicidad a lo largo de $L^{\prime}$ (Proposición 2), se sigue que:

$$
m_{0}(Z)=m_{0}\left(Z^{\prime}, L^{\prime}\right)=m_{0}\left(Z^{\prime}\right) i_{\tilde{p}^{\prime}}\left(\dot{E}^{-1}(0), \tilde{L}^{\prime}\right)
$$

Por lo tanto $m_{0}(Z)$ es divisible por $m_{0}\left(Z^{\prime}\right)$. Análogamente. si $\tilde{q}^{\prime} \in$ $E^{-1}(0)-S^{\prime}$, donde $S^{\prime}$ es la hipersuperficie de tangencia de $\tilde{\mathcal{F}_{Z^{\prime}}}$, tenemos que $m_{0}\left(Z^{\prime}\right)$ es divisible por $m_{0}(Z)$ y por lo tanto $m_{0}(Z)=m_{0}\left(Z^{\prime}\right)$.

Más aún, si $\tilde{p} \in E^{-1}(0) \cap S$, de la Proposición 2 y del Lema anterior. tenemos que:

$$
i_{\tilde{p}}\left(E^{-1}(0), \tilde{L}\right)=\frac{m_{0}(Z, L)}{m_{0}(Z)}=\frac{m_{0}\left(Z^{\prime}, L^{\prime}\right)}{m_{0}\left(Z^{\prime}\right)}=i_{\tilde{p}^{\prime}}\left(E^{-1}(0), \tilde{L}^{\prime}\right) .
$$

De esta manera, hemos demostrado nuestro resultado principal:

Teorema. La multiplicidad algebraica de una foliación de $\mathcal{D}_{0}^{n}$ y el indice de intersección, son invariantes topológicos. Más especificament.e, sean $\mathcal{F}_{Z}, \mathcal{F}_{Z^{\prime}} \in \mathcal{D}_{0}^{n}, \tilde{p}, \tilde{p}^{\prime}, \tilde{L}$ y $\tilde{L}^{\prime}$ como antes. Si $\mathcal{F}_{Z} \sim_{\text {top }} \mathcal{F}_{Z^{\prime}}$ entonces

1. $m_{0}(Z)=m_{0}\left(Z^{\prime}\right)$

2. $i_{\tilde{p}}\left(E^{-1}(0), \tilde{L}\right)=i_{\tilde{p}^{\prime}}\left(E^{-1}(0), \tilde{L}^{\prime}\right)$.

\section{BIBLIOGRAFÍA}

[1] R. Benazic, Caracterización de singularidades dicríticas en foliaciones de dimension uno, PESQUIMAT, Vol. 1, N 1, (1998), p. 73-81.

[2] R. Benazic, Sobre el Número de Milnor, PESQUIMAT, Vol. III, N 1, (2000). p. $1-15$. 
[3] C. Camacho, L. Neto, P. Sad, Topological Invariants and Equidesingularization for Holomorphic Vector Fields, J. Differential Geometry 20, (1984), p. 143-174.

[4] D. Cerveau, J. Mattei, Formes Intégrables Holomorphes Singulières, Asterisque, $97,(1982)$.

[5] E. Chirka, Complex Analytic Sets, MIA, Kluwer Academic Publishers. Dordrecht, Boston, London (1989).

[6] P. Griffiths, J. Harris, Principles of Algebraic Geometry, Wilev-Intersciencic. New York, (1978).

[7] R. Gunning, H. Rossi, Analytic functions of several complex variables, PrenticeHall, Englewood Cliffs, NJ., (1965).

[8] J. Mattei, R. Moussu, Holonomie et intégrales premières, Ann. Sci. Ecole. Norm. Sup. (4) 13 (1980) p. 469-523.

[9] H. Whitney, Complex Analytic Varieties, Addison-Wesley Publishing Company, (1972). 